\title{
A Eukaryotic Specific Transmembrane Segment is Required for Tetramerization in AMPA Receptors
}

\author{
Catherine L. Salussolia, ${ }^{1,2,4}$ Quan Gan, ${ }^{1,4}$ Rashek Kazi, ${ }^{1,2,4}$ Puja Singh, ${ }^{5}$ Janet Allopenna, ${ }^{4}$ Hiro Furukawa, ${ }^{5}$ \\ and Lonnie P. Wollmuth ${ }^{1,3,4}$ \\ ${ }^{1}$ Graduate Program in Neuroscience, ${ }^{2}$ Medical Scientist Training Program, ${ }^{3}$ Department of Neurobiology and Behavior, and ${ }^{4}$ Center for Nervous System \\ Disorders, Stony Brook University, Stony Brook, New York 11794-5230, and ${ }^{5}$ W.M. Keck Structural Biology Laboratory, Cold Spring Harbor Laboratories, \\ Cold Spring Harbor, New York 11724
}

\begin{abstract}
Most fast excitatory synaptic transmission in the nervous system is mediated by glutamate acting through ionotropic glutamate receptors (iGluRs). iGluRs (AMPA, kainate, and NMDA receptor subtypes) are tetrameric assemblies, formed as a dimer of dimers. Still, the mechanism underlying tetramerization-the necessary step for the formation of functional receptors that can be inserted into the plasma membrane-is unknown. All eukaryotic compared to prokaryotic iGluR subunits have an additional transmembrane segment, the M4 segment, which positions the physiologically critical C-terminal domain on the cytoplasmic side of the membrane. AMPA receptor (AMPAR) subunits lacking M4 do not express on the plasma membrane. Here, we show that these constructs are retained in the endoplasmic reticulum, the major cellular compartment mediating protein oligomerization. Using approaches to assay the native oligomeric state of AMPAR subunits, we find that subunits lacking M4 or containing single amino acid substitutions along an "interacting" face of the M4 helix that block surface expression no longer tetramerize in either homomeric or heteromeric assemblies. In contrast, subunit dimerization appears to be largely intact. These experiments define the M4 segment as a unique functional unit in AMPARs that is required for the critical dimer-to-tetramer transition.
\end{abstract}

\section{Introduction}

Dynamic regulation of iGluR expression at the plasma membrane directly modulates synaptic strength, membrane excitability, and neuronal network activity (Citri and Malenka, 2008; Anggono and Huganir, 2012). Biogenesis of iGluRs - the transcription, translation, folding, and oligomerization of iGluR subunits to form functional tetrameric assemblies-are key checkpoints regulating the availability of iGluRs for plasma membrane insertion. iGluR tetramers assemble as a dimer of dimers (Stephenson et al., 2008; Mayer, 2011). The extracellularly located amino-terminal domain (ATD) mediates dimerization in a subfamily-specific manner (Mayer, 2011). Additionally, various mechanisms including mRNA editing and alternative splicing modulate the assembly of tetramers (Nakagawa, 2010; Sukumaran et al., 2012). However, the determinants of iGluR tetramerization are unknown.

\footnotetext{
Received May 31, 2012; revised April 29, 2013; accepted May 3, 2013.

Author contributions: C.L.S., Q.G., H.F., and L.P.W. designed research; C.L.S., Q.G., R.K., P.S., J.A., and H.F. performed research; C.L.S., Q.G., R.K., P.S., H.F., and L.P.W. analyzed data; C.L.S., Q.G., R.K., H.F., and L.P.W. wrote the paper.

This work was supported by NIMH R01 Grants MH066892 (L.P.W.) and MH085926 (H.F.), the Robertson Research Fund of Cold Spring Harbor Laboratory (H.F.), an SBU-CSHL collaborative grant (H.F., L.P.W.), and NINDS National Research Service Award NS073382 (C.L.S.). We thank Drs. Myles Akabas, David Talmage, and Maurice Kernan for helpful discussions and/or comments on this manuscript. We thank Dr. Nami Tajima for technical assistance. We thank Dr. Morgan Sheng for supplying the HA-tagged GluA2 subunit.

Correspondence should be addressed to Dr. Lonnie P. Wollmuth, Department of Neurobiology and Behavior, Center for Nervous System Disorders, State University of New York at Stony Brook, Stony Brook, NY 11794-5230. E-mail: lonnie.wollmuth@stonybrook.edu.

DOI:10.1523/JNEUROSCI.2626-12.2013

Copyright $\odot 2013$ the authors $\quad 0270-6474 / 13 / 339840-06 \$ 15.00 / 0$
}

In iGluRs, the core of the ion channel, formed by the M1 and M3 transmembrane segments and an intracellular M2 loop, is structurally and evolutionarily related to an inverted twotransmembrane $\mathrm{K}^{+}$channel (Wo and Oswald, 1995). In twoas well as six-transmembrane $\mathrm{K}^{+}$channels, the amino- and carboxy-termini are located intracellularly and are required for tetramerization (Deutsch, 2002). In contrast, the intracellular C-terminal domain (CTD) in iGluRs is essential for regulating their trafficking and localization (Anggono and Huganir, 2012), but its complete removal does not prevent the formation of functional, tetrameric NMDA (Puddifoot et al., 2009) or AMPA (Salussolia et al., 2011) receptors. Similarly, iGluR tetramerization still occurs in the absence of the ATD (Pasternack et al., 2002; Schüler et al., 2008). Furthermore, the mammalian iGluR ligandbinding domain (LBD), composed of the discontinuous S1 and S2 polypeptides, does not readily form dimers in solution (Sun et al., 2002), suggesting that it does not substantially contribute to oligomerization. Thus, other structural elements beyond the ATD, LBD, and CTD must be involved in iGluR tetramerization.

Like two-transmembrane $\mathrm{K}^{+}$channels, prokaryotic iGluR subunits (i.e., GluR0) have only two-transmembrane segments and form functional receptors expressed at the plasma membrane (Chen et al., 1999). In contrast, all known eukaryotic iGluR subunits contain an additional transmembrane segment, the M4 segment, C-terminal to the ion channel core. The M4 segment positions the CTD intracellularly, permitting its interaction with intracellular signaling pathways and postsynaptic density proteins. Furthermore, mammalian iGluR subunits require M4 for surface expression in NMDA receptors (NMDARs) (Horak et al., 
2008, but see Meddows et al., 2001) and AMPA receptors (AMPARs) (Salussolia et al., 2011). Recently, using a tryptophan substitution screen, we identified a specific face of M4 that prevented iGluR surface expression (Salussolia et al., 2011). Given that the specific M4 face aligns closely with the M1 and M3 transmembrane segments of an adjacent subunit (Sobolevsky et al., 2009), we hypothesized that the M4 segment may mediate AMPAR assembly.

Here, we find that AMPAR subunits lacking M4 or containing single amino acid substitutions in the specific M4 face no longer form tetrameric complexes. These experiments highlight an essential and previously unrecognized role of the M4 segment in the assembly of AMPARs.

\section{Materials and Methods}

Mutagenesis and expression. Site-directed mutations were made in and around the M4 transmembrane segments of rat AMPAR subunits all in the "flip" form: GluAl (accession \#P19490) and the unedited (Q) and edited (R) forms of GluA2 (accession \#P19491). For certain experiments and where indicated in the text, subunits were tagged either with hemagglutinin (HA, inserted between the ATD and S1) (Man et al., 2000) or GFP ( $\mathrm{C}$ terminal). All mutations were generated using PCR-based methods and confirmed by sequence analysis. Numbering is for the mature protein with signal peptides of 18 (GluA1) and 21 (GluA2). AMPAR subunits were expressed in human embryonic kidney (HEK) 293 cells (Salussolia et al., 2011).

Immunocytochemistry. HEK 293 cells were plated at a density of $0.5 \times$ $10^{6}$ on coverslips pretreated with nitric acid and coated with poly-Dlysine. Cells were maintained in $10 \% \mathrm{FBS}$ at $37^{\circ} \mathrm{C}$ and $95 \% \mathrm{O}_{2} / 5 \% \mathrm{CO}_{2}$. Surface expression was determined as described previously (Salussolia et al., 2011).

To determine subcellular localization of HA-tagged AMPAR constructs, cells were fixed in $4 \%$ paraformaldehyde, permeabilized with $0.25 \%$ Triton X-100, and blocked in $1 \%$ goat serum. Primary antibodies labeling the endoplasmic reticulum (calreticulin; Abcam; AB22683), or Golgi bodies (GM130; BD Biosciences; 610822), along with anti-HA (Covance; MMS-101P; mouse monoclonal 16B12) were added directly onto the coverslip and incubated in a humidified chamber at $37^{\circ} \mathrm{C}$ for $1 \mathrm{~h}$. Cells were rinsed with PBS and incubated with Alexa Fluor 546 goat anti-mouse and Alexa Fluor 488 goat anti-rabbit secondary antibodies (Invitrogen) at room temperature. Cells were examined using an upright Fluoview FV1000 confocal microscope (Olympus).

Blue native-PAGE. Cells were rinsed in PBS, harvested in solubilization buffer [ $2 \% N$-dodecyl- $\alpha$-D-maltopyranoside (DDM; Affymetrix; D310HA) dissolved in PBS containing protease inhibitors (Thermo Scientific; 1861278)], rotated for $1 \mathrm{~h}$ at $4^{\circ} \mathrm{C}$, and centrifuged at $50 \mathrm{k}$ RPM (Beckman TLA 120.2 rotor) for $40 \mathrm{~min}$ at $4^{\circ} \mathrm{C}$. Membrane proteins contained in the supernatant were resolved using the commercially available Invitrogen blue native (BN)-PAGE system. Briefly, protein samples mixed with $1 \times$ NativePAGE sample buffer and 0.05\% NativePAGE G-250 additive were loaded onto Novex $4-16 \%$ Bis/Tris gradient gels. Proteins were separated at constant voltage at $4^{\circ} \mathrm{C}$. Gels were transferred overnight at constant amperage to polyvinylidene fluoride membranes. Following transfer, membranes were briefly rinsed with methanol, and protein transfer was confirmed by Ponceau staining. Membranes were then rinsed with water, rehydrated with methanol, and rinsed with TBS-T (0.05\% Tween 20$)$ before block in 5\% milk-TBS. Membranes were incubated with either anti-GluA1 (Millipore; MAB2269; mouse monoclonal RH95) or anti-GluA2 (Millipore; MAB397; mouse monoclonal 6C4) (both targeting the ATD) and anti-mouse IgG secondary antibodies (Santa Cruz Biotechnologies; sc-2030). Blots were developed using luminol reagent (Santa Cruz Biotechnologies; sc-2048) before exposure to chemiluminescence blue-sensitive film (Crystalgen). In certain instances, lanes from the same gel are presented in a different order from the original gel (indicated by a thin space between lanes) for clarity of presentation.

Fluorescence-detection size exclusion chromatography. Cells were transfected with GluA1 or GluA2(Q) tagged at the C terminal with GFP. Cells
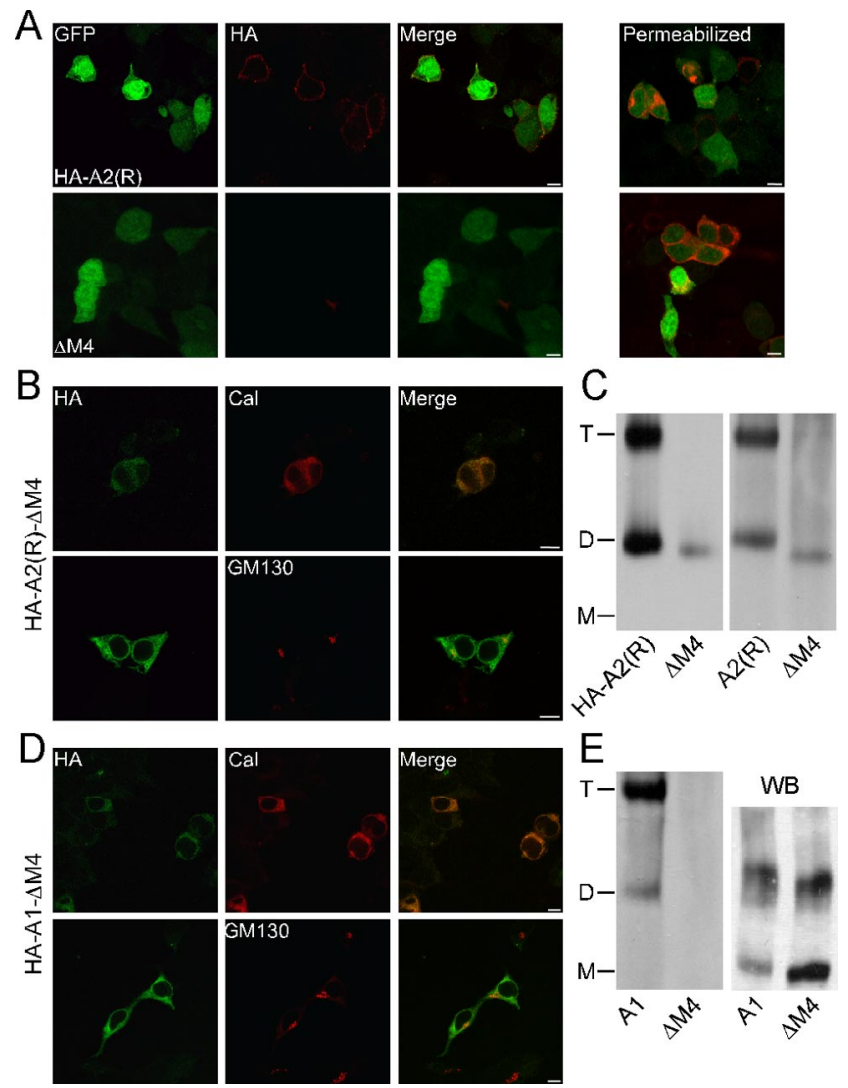

Figure 1. Deletion of the M4 segment disrupts AMPAR assembly. A, Immunocytochemistry (ICC) of HEK 293 cells cotransfected with eGFP and HA-tagged wild-type GluA2(R) [HA-A2(R)] or a GluA2(R) construct with a stop codon introduced before $M 4(\Delta M 4)$ under nonpermeabilized (left) or permeabilized (right) conditions. Protein expression was detected using an anti-HA antibody and Alexa Fluor 633 secondary antibody. $\boldsymbol{B}, \boldsymbol{D}$, ICC of HA-GluA2(R)- $\Delta \mathrm{M} 4$ (B) or HAGluA1- $\Delta M 4$ (D) under permeabilized conditions. Cells were labeled with anti-HA and either calreticulin (top), an ER marker, or GM130 (bottom), a Golgi marker. C, BN-PAGE of HA-tagged (left two lanes) or untagged (right two lanes) wild type or $\triangle M 4$ GluA2(R). The approximate location of the tetramer (T), dimer (D) and monomer (M) bands were identified using Apoferritin (Sigma) and NativeMark (Invitrogen) markers. E, BN-PAGE (left two lanes) or Western blot (WB; right two lanes) of wild type or $\Delta \mathrm{M} 4$ GluA1. Scale bars: $10 \mu \mathrm{m}$.

were rinsed with PBS, pelleted, and resuspended in $300 \mu$ l solubilization buffer containing $20 \mathrm{~mm}$ Tris- $\mathrm{HCl}, \mathrm{pH}, 200 \mathrm{~mm} \mathrm{NaCl}$ (TBS) supplemented with $1 \%$ DDM (Affymetrix), and $1 \mathrm{~mm}$ phenylmethylsulphonyl fluoride. Cells were lysed using a Misonix Sonicator 3000 (4 times, 30 s, power level 2) and rotated for $1 \mathrm{~h}$ at $4^{\circ} \mathrm{C}$ before ultracentrifugation (TLA110 rotor) at $70,000 \mathrm{rpm}$ for $10 \mathrm{~min}$. A fraction of supernatant $(100 \mu \mathrm{l})$ was loaded onto a Superose 6 column (10/300 GL; GE Healthcare), preequilibrated with TBS buffer containing $0.05 \% \mathrm{DDM}$, and run at a flow rate of $0.4 \mathrm{ml} / \mathrm{min}$. The eluent from Superose 6 column was passed through a fluorometer with the following settings: excitation, $475 \mathrm{~nm}$; emission, $507 \mathrm{~nm}$; time increment, $0.5 \mathrm{~s}$; integration time, $1 \mathrm{~s}$; recording time, $0-4500 \mathrm{~s}$. Fractionations were collected and chromatograms for a given transfection cycle were normalized to the tetramer peak (occurring between 1700 and 2000 s) for wild types and were plotted using Igor Pro (Wavemetrics).

Whole-cell current recordings and data analysis. For whole-cell recordings, cells were cotransfected with cDNA for each GluA1, GluA2(R), and a vector for enhanced GFP at a ratio (in micrograms) of 4.5:4.5:1. Recordings were made $24-72 \mathrm{~h}$ after transfection.

Currents were recorded at room temperature $\left(20-23^{\circ} \mathrm{C}\right)$ using an EPC-9 amplifier with Patchmaster software (HEKA Elektronik), lowpass filtered at $5 \mathrm{kHz}$, and digitized at $20 \mathrm{kHz}$. External solutions were applied using a piezo-driven double-barrel application system (Yelshansky et al., 2004). The pipette solution consisted of the following (in mM): $140 \mathrm{KCl}, 10 \mathrm{HEPES}$, and 1 BAPTA, pH $7.2(\mathrm{KOH})$. The external 
solution consisted of the following (in $\mathrm{mm}$ ): $140 \mathrm{NaCl}, 1.8 \mathrm{CaCl}_{2}, 1 \mathrm{MgCl}_{2}$, and 10 HEPES, pH $7.2(\mathrm{NaOH})$. All solutions also contained cyclothiazide ( $30 \mu \mathrm{M})$ to minimize desensitization. Analysis was performed using Igor Pro.

\section{Results}

Removal of the M4 segment disrupts tetramerization in AMPAR subunits To address the potential role of the M4 segment in iGluR biogenesis, we initially used the edited form of the GluA2 subunit [GluA2(R)] because of its broad distribution in the nervous system. A HA-tagged wild-type GluA2(R) subunit [HA-GluA2(R)] expressed in HEK 293 cells showed detectable surface expression (Fig. 1A, top) and glutamate-activated currents (data not shown). As expected for a construct that forms functional/surface-expressing receptors, HA-GluA2(R) (Fig. 1C, left) and untagged GluA2(R) (right) yielded a tetramer band, as well as a dimer band, when analyzed under nonreducing and nondenaturing conditions by BN-PAGE.

GluA2(R) subunits lacking the M4 segment [HA-GluA2(R)- $\Delta$ M4], like similar constructs for NMDA (Horak et al., 2008) and AMPA (GluA1) (Salussolia et al., 2011) receptor subunits, did not show detectable surface expression (Fig. $1 A$, bottom). Protein maturation and subunit oligomerization occur in the endoplasmic reticulum (ER). Upon tetramerization and passing quality control mechanisms, iGluRs are exported to the Golgi complex before insertion in the plasma membrane (Fleck, 2006). To determine where in this secretory pathway the $\Delta \mathrm{M} 4$ construct is retained, we visualized permeabilized cells probed with $\mathrm{HA}$ as well as antibodies specific for the ER (calreticulin) or the Golgi complex (GM130) (Fig. 1B). The $\Delta \mathrm{M} 4$ construct colocalized with the ER but not the Golgi marker, suggesting that it was retained in the ER possibly in an immature oligomeric form. Consistent with this idea, the HA-tagged (Fig. 1C, left) and untagged (right) GluA2(R)- $\Delta \mathrm{M} 4$ did not show a tetramer band when resolved by BN-PAGE, but rather only a dimer band.

We also performed similar experiments on GluA1- $\Delta \mathrm{M} 4$, whose lack of surface expression has been characterized extensively (Salussolia et al., 2011). Like HA-GluA2(R)- $\Delta$ M4, GluA1- $\Delta$ M4 localizes to the ER (Fig. 1D, HA-tagged) and shows no tetramer band by BN-PAGE (Fig. 1E, untagged). However, GluA1- $\Delta$ M4 also does not show a detectable dimer or monomer band presumably due to aggregate forma-

C

$\mathrm{F}-\mathrm{GluA1}$
A M4 Segment B
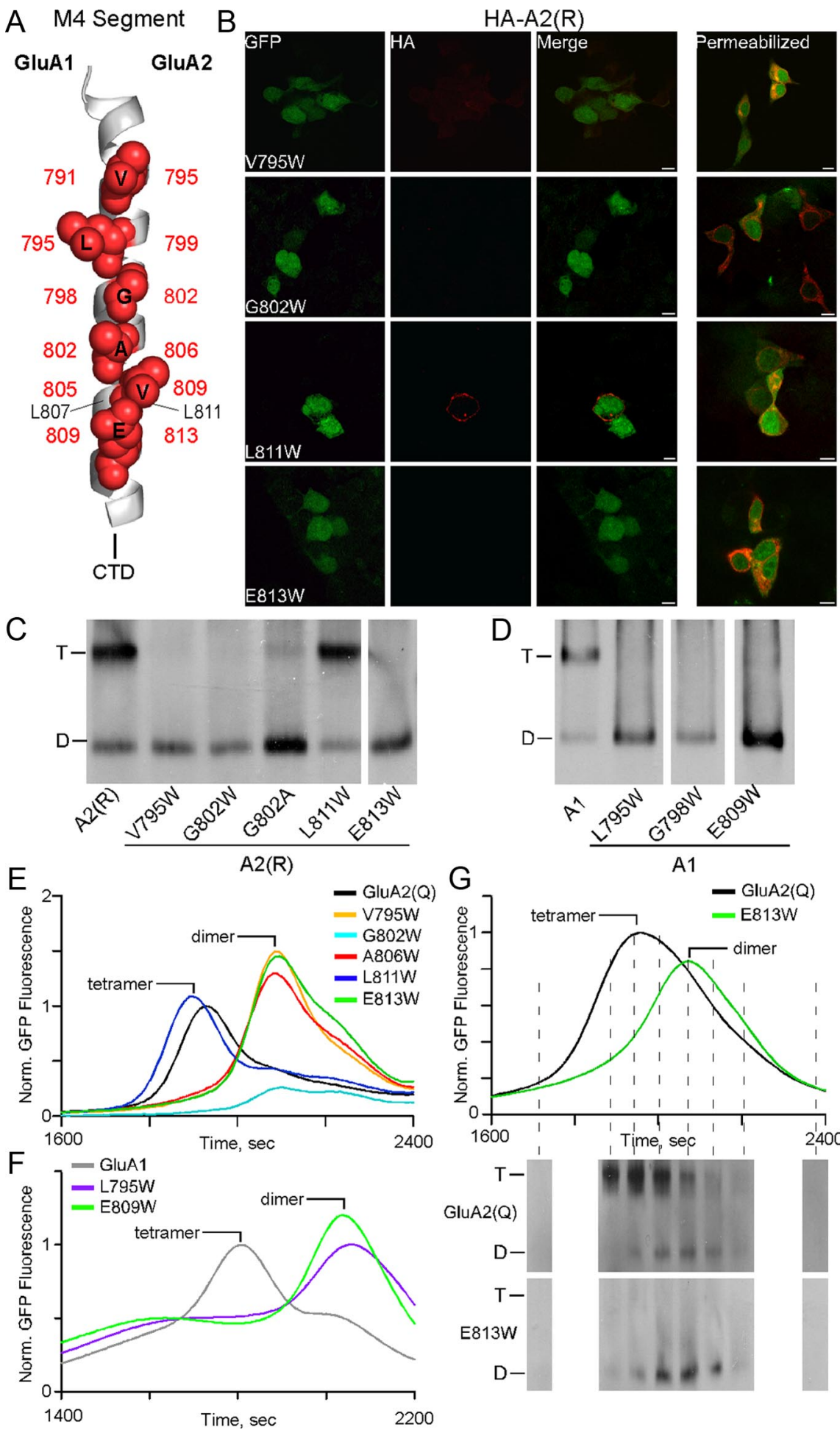

Figure 2. Substitutions of the interacting M4 face disrupt tetramerization. $\boldsymbol{A}$, A highlighted (red) face of the M4 segment (PDB ID 3KG2) (Sobolevsky et al., 2009): valine-leucine-glycine-alanine-valine-glutamate. Numbering for GluA1 (left) and GluA2 (right) is for the mature protein. GluA1 subunits containing tryptophan substitutions of these positions do not show surface expression, whereas those containing L807W showed membrane currents (Salussolia et al., 2011). B, Immunocytochemistry of HA-tagged tryptophan-substituted GluA2(R) subunits. Scale bar, $10 \mu \mathrm{m}$. C, D, BN-PAGE of tryptophan-substituted GluA2(R) (C) or GluA1 (D) subunits. $\boldsymbol{E}, \boldsymbol{F}$, FSEC of wild-type or tryptophan-substituted GluA2 $(Q)(\boldsymbol{E})$ or GluA1 $(\boldsymbol{F})$ subunits. $\boldsymbol{G}$, Top, FSEC of wild-type GluA2(Q) or GluA2(Q) (E813W). Bottom, BN-PAGE of fractions (times point indicated by dashed lines) from FSEC.

tion since the protein is not degraded as assayed by nonreducing Western immunoblots (Fig. 1E, right). Hence, in the absence of the M4 segment, AMPAR subunits do not form tetramers. 

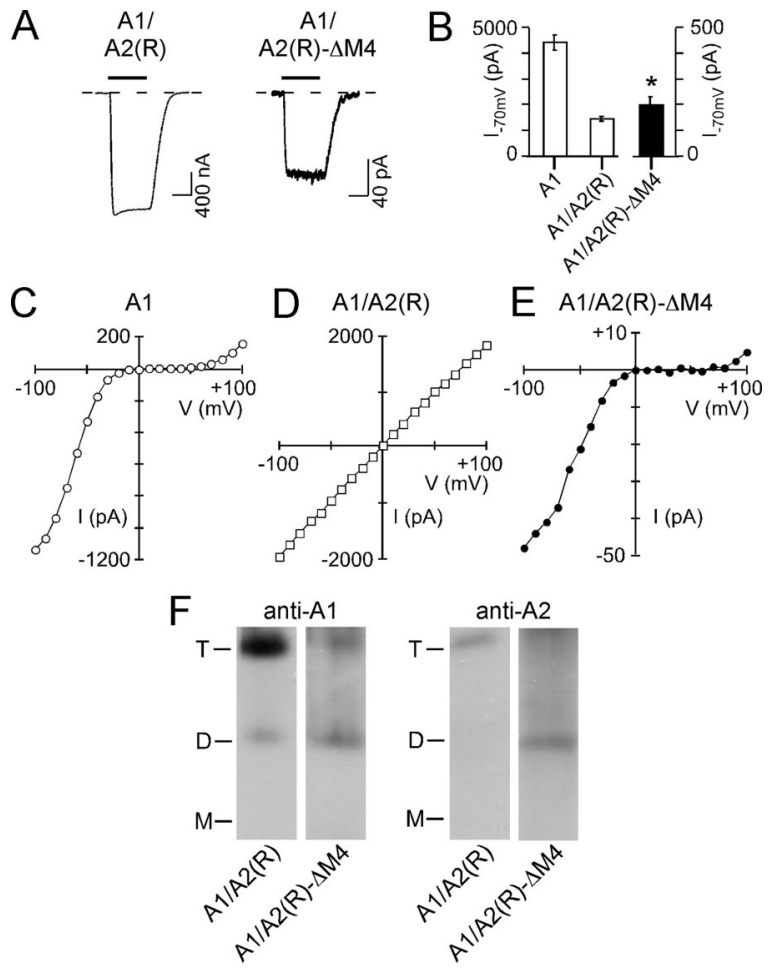

Figure 3. The M4 segment in heteromeric AMPAR assembly. $A$, Whole-cell currents at -70 $m V$ in HEK 293 cells transfected with either GluA1/GluA2(R) or GluA1/GluA2(R)- $\Delta$ M4 (1:1 ratios). Solid bar indicates glutamate $(3 \mathrm{~mm}$ ) application. $\boldsymbol{B}$, Mean current amplitudes ( \pm SEM; $n>5$ ). ${ }^{*} p<0.05$ [values significantly different from GluA1/GluA2(R); Student's $t$ test]. $C-E$, Current-voltage relationships $(-100$ to $+100 \mathrm{mV})$ for HEK 293 cells transfected with indicted CDNA. $\boldsymbol{F}$, BN-PAGE of transfected HEK 293 cells probed with either anti-A1 (left) or anti-A2 (right).

\section{Substitutions of the interacting M4 face disrupt tetramerization}

The $\Delta \mathrm{M} 4$ manipulation is limited in terms of addressing the structural-functional significance of the M4 segment due to its all-or-none nature. We therefore focused on a previously identified "interacting" face of the M4 helix represented by positions valine $(V)$, leucine $(\mathrm{L})$, glycine $(\mathrm{G})$, alanine $(\mathrm{A})$, valine $(\mathrm{V})$, and glutamate (E) (Fig. 2A, red positions). Tryptophan (W) has a large, bulky side chain that can disrupt helix-helix interactions. Tryptophan substitutions of these positions in GluA1 as well as several adjacent positions, but not of those located on the opposite side of the helix in contact with lipid including L807, prevented surface expression of AMPARs (Salussolia et al., 2011) presumably in a manner comparable to $\Delta \mathrm{M} 4$. This M4 face is termed "interacting" because it aligns closely with M1 and M3 segments of an adjacent subunit (Sobolevsky et al., 2009).

In terms of surface expression, HA-GluA2(R) subunits containing single M4 tryptophan substitutions (Fig. 2B) paralleled previously published expression patterns for homologous GluA1 constructs (Salussolia et al., 2011). Specifically, like wild type (Fig. 1A), GluA2(L811W), homologous to the functional GluA1(L807W), showed detectable surface expression (Fig. 2B) and prominent tetramer and dimer bands by BN-PAGE (Fig. $2 C)$. Conversely, GluA2(R) subunits containing substitutions of the M4 interacting face (V795W, G802W, or E813W) did not show detectable surface expression (Fig. $2 B$, nonpermeabilized), though they did show protein expression (Fig. $2 B$, permeabilized). These substitutions of the M4 interacting face, like the $\Delta \mathrm{M} 4$ construct, not only disrupt surface expression, but also pre- vent the formation of tetramers, showing only dimer bands by BN-PAGE (Fig. 2C). A less dramatic alanine substitution at G802 (G802A) also significantly reduced tetramerization (Fig. 2C). As in GluA2(R), tryptophan substitutions in GluA1 blocked tetramerization (Fig. 2D).

As an additional approach to address the oligomeric state of iGluR subunits, we took advantage of fluorescence-detection size exclusion chromatography (FSEC) (Kawate and Gouaux, 2006). For these experiments, we initially used the unedited (Q) form of the GluA2 subunit containing a C-terminal GFP tag because GluA2(Q) provides a well-defined oligomeric profile (Sobolevsky et al., 2009). FSEC also allows for the identification of misfolded proteins, which elute as higher-order aggregates (Kawate and Gouaux, 2006). As expected, surface-expressing wildtype GluA2(Q) and L811W showed prominent tetramer peaks (Fig. 2D). In contrast, subunits containing substitutions of the interacting face (V795W, G802W, A806W, or E813W) show almost exclusively dimer peaks. The chromatograms also did not show higher-order oligomeric peaks, suggesting that the various constructs do not undergo extensive protein misfolding or aggregation. Furthermore, BN-PAGE on fractions from FSEC for GluA2(Q) and E813W (Fig. 2G) verify that the peaks in the chromatogram correspond to tetramers and dimers.

GluA2(Q) is most likely not expressed in the nervous system (Traynelis et al., 2010). We therefore generated a GFP C-terminally tagged GluA1 subunit. Although the oligomeric peaks in the chromatogram were less distinct than those for GluA2(Q), wild-type GluA1 subunits existed predominantly as tetramers (Fig. $2 F$ ). In contrast, subunits containing tryptophan substitutions of the interacting face, L795 or E809, show only dimer peaks (Fig. $2 F$ ). Thus, single substitutions of the interacting face that disrupt surface expression in homomeric GluAl and GluA2 do so by preventing the dimer-to-tetramer transition, recapitulating the oligomeric phenotype of the $\Delta \mathrm{M} 4$ construct.

\section{The M4 segment mediates tetramerization in heteromeric AMPARs}

AMPAR subunits can form functional homotetramers. However, native AMPARs are almost always heterotetramers, often composed of GluA1 and GluA2(R) (Lu et al., 2009). To test the role of M4 in heterotetramer formation, we initially assayed membrane expression using glutamate-activated currents in HEK 293 cells. Expression of GluA1 alone $(-4410 \pm 310 \mathrm{pA}$, mean \pm SEM; $n=9$ recordings) or together with GluA2(R) (Fig. 3A; $-1440 \pm 100 \mathrm{pA}, n=6$ ) yields large membrane currents (Fig. $3 B)$. GluA2(R)- $\Delta \mathrm{M} 4$ expressed alone did not show any detectable glutamate-activated currents (data not shown). However, when GluA2(R)- $\Delta$ M4 was coexpressed with GluA1 (Fig. $3 A$ ), detectable currents were observed, but their magnitude $(-200 \pm 30 \mathrm{pA}, n=6$; Fig. $3 B$, note the changed axis scale) was significantly less than that for GluA1/GluA2(R).

The small currents detected for GluA1/GluA2(R)- $\Delta \mathrm{M} 4$ could reflect either heteromeric receptors that gate poorly or homomeric GluA1 receptors not incorporated into a heteromeric assembly. To test these alternatives, we characterized the voltage dependence of currents in HEK 293 cells transfected with various AMPAR subunits (Fig. 3C-E). As expected, the current-voltage $(I-V)$ relationship for homomeric GluA1 shows a characteristic double rectification (Fig. $3 C$ ), whereas it is largely linear when GluA1 is coexpressed with GluA2(R) (Fig. 3D) (Traynelis et al., 2010). Notably, currents detected in cells transfected with GluA1/ GluA2(R)- $\Delta$ M4 (Fig. $3 E$ ) display a doubly rectifying $I-V$ relationship indistinguishable from that of homomeric GluA1. Thus, 
the small current amplitudes detected in GluA1/GluA2(R)- $\Delta \mathrm{M} 4$ are most likely mediated by homomeric GluA1 receptors, suggesting that heteromeric receptors containing GluA2(R)- $\Delta \mathrm{M} 4$ and GluA1 cannot access the plasma membrane, presumably because tetramerization is blocked.

Notably, the current amplitudes for the apparent homomeric GluA1 receptors $(<200 \mathrm{pA})$ when GluA1 is cotransfected with GluA2(R)- $\Delta \mathrm{M} 4$ are significantly smaller than when GluA1 is transfected alone (approximately $-4400 \mathrm{pA}$ ). One explanation is that the process of heterodimerization, which is driven by the ATD (Rossmann et al., 2011), between GluA1 and GluA2(R)$\Delta \mathrm{M} 4$ remains intact. These heterodimers cannot tetramerize, but act as a sink for GluA1 subunits, resulting in a significantly decreased membrane expression of homomeric GluA1 receptors. Results for homomeric subunits with M4 manipulations where dimerization remains intact (Fig. 1) are consistent with this latter explanation.

To further address the contribution of M4 to heterotetramerization, we tested the oligomeric state of constructs using BNPAGE (Fig. 3F). For GluA1/GluA2(R) [A1/A2(R)], tetramer bands were prominent, especially when compared to dimer bands, when probed with either anti-GluA1 (left) or anti-GluA2 (right). In contrast, for GluA1/GluA2(R)- $\Delta \mathrm{M} 4$, the dimer band was significantly more prominent than the tetramer band, consistent with M4 being required for heterotetrameric assembly. Furthermore, a tetramer band could be detected for A1/A2(R)$\Delta \mathrm{M} 4$ when probed with anti-GluA1. These tetramer bands presumably reflect homomeric GluA1 receptors. Overall, these as well as the electrophysiological results are consistent with a role of the M4 segment in heterotetrameric assembly.

\section{Discussion}

Tetramerization of iGluR subunits is the necessary step to yield functional iGluR complexes that can be inserted into the plasma membrane. Our experiments demonstrate that in AMPARs, the eukaryotic-specific M4 segment is required for tetramerization in both homomeric and heteromeric receptors. AMPAR subunits lacking M4, GluA2(R)- $\Delta \mathrm{M} 4$, or GluA1- $\Delta \mathrm{M} 4$ do not tetramerize (Figs. $1 C, E, 3$ ) and, consistent with their immature oligomeric status, are retained in the ER (Figs. $1 B, D$ ). Additionally, GluA1 or GluA2(R) subunits containing mutations that disrupt helixhelix interactions along a specific face of the M $4 \alpha$-helix (Fig. 2A), a face that aligns with M1 and M3 of an adjacent subunit (Sobolevsky et al., 2009), also do not form tetramers as assayed by BN-PAGE (Fig. $2 C, D$ ) and/or by FSEC (Fig. $2 E, F$ ). Finally, manipulations that disrupted tetramerization did not prevent dimerization, suggesting that the initial step of oligomerization is largely intact. Thus, the M4 segment-and its presumed interaction with the adjacent M1 and M3 segments-is required for the dimer-to-tetramer transition in homomeric as well as heteromeric AMPARs.

Like AMPARs, the M4 segment may be required for tetramerization in other iGluR subtypes. NMDARs are obligate heteromers typically composed of two GluN1 and two GluN2 subunits. NMDARs are presumably assembled as dimer of heterodimers (Furukawa et al., 2005; Karakas et al., 2011; Lee and Gouaux, 2011). The ubiquitous GluN1 subunit may act as a substrate for dimer formation (Atlason et al., 2007), but at present it remains uncertain whether such homodimers exist in vivo (Papadakis et al., 2004; Farina et al., 2011). The deletion of the GluN1 M4 segment either blocked (Horak et al., 2008) or permitted (Meddows et al., 2001) surface expression of NMDARs. However, previous experiments have suggested that for NMDAR subunits, the ion channel core (M1-M3) is required for homodimer formation, whereas both the ion channel core and the M4 segment (as well as S2) are necessary for heterotetrameric assembly (Cao et al., 2011). Part of the uncertainty concerning the role of the M4 segment in NMDAR assembly may reflect that it is not required for dimerization, but only tetramerization. Nevertheless, specific experiments are needed to directly test the role of the M4 segment in tetramerization in NMDA as well as kainate receptors.

In the ER there are apparently dimer pools of AMPA (Sukumaran et al., 2012) and NMDA (Huh and Wenthold, 1999) receptor subunits, suggesting that the formation of tetrameric receptors from these dimers might be a regulated step. Numerous intracellular signaling pathways driven by synaptic activity interact with the CTD, which is connected to M4 to increase the forward trafficking of AMPARs (Anggono and Huganir, 2012; Lu and Roche, 2012). Since forward trafficking depends on available pools of tetrameric iGluRs, one intriguing possibility is that intracellular signaling molecules can also enhance iGluR availability via $\mathrm{M} 4$-mediated tetramerization.

Manipulations of the M4 segment do not seem to dramatically affect dimerization, the initial step in iGluR oligomerization, which is largely mediated by the extracellularly located ATDs (Mayer, 2011). On the other hand, our results indicate that tetramerization is largely driven by transmembrane segment interactions, as has been suggested previously (Ayalon and Stern-Bach, 2001; Cao et al., 2011).

\section{References}

Anggono V, Huganir RL (2012) Regulation of AMPA receptor trafficking and synaptic plasticity. Curr Opin Neurobiol 22:461-469. CrossRef Medline

Atlason PT, Garside ML, Meddows E, Whiting P, McIlhinney RA (2007) N-Methyl-D-aspartate (NMDA) receptor subunit NR1 forms the substrate for oligomeric assembly of the NMDA receptor. J Biol Chem 282: 25299-25307. CrossRef Medline

Ayalon G, Stern-Bach Y (2001) Functional assembly of AMPA and kainate receptors is mediated by several discrete protein-protein interactions. Neuron 31:103-113. CrossRef Medline

Cao JY, Qiu S, Zhang J, Wang JJ, Zhang XM, Luo JH (2011) Transmembrane region of N-methyl-D-aspartate receptor (NMDAR) subunit is required for receptor subunit assembly. J Biol Chem 286:27698-27705. CrossRef Medline

Chen GQ, Cui C, Mayer ML, Gouaux E (1999) Functional characterization of a potassium-selective prokaryotic glutamate receptor. Nature 402:817821. CrossRef Medline

Citri A, Malenka RC (2008) Synaptic plasticity: multiple forms, functions, and mechanisms. Neuropsychopharmacology 33:18-41. CrossRef Medline

Deutsch C (2002) Potassium channel ontogeny. Annu Rev Physiol 64: 19-46. CrossRef Medline

Farina AN, Blain KY, Maruo T, Kwiatkowski W, Choe S, Nakagawa T (2011) Separation of domain contacts is required for heterotetrameric assembly of functional NMDA receptors. J Neurosci 31:3565-3579. CrossRef Medline

Fleck MW (2006) Glutamate receptors and endoplasmic reticulum quality control: looking beneath the surface. Neuroscientist 12:232-244. CrossRef Medline

Furukawa H, Singh SK, Mancusso R, Gouaux E (2005) Subunit arrangement and function in NMDA receptors. Nature 438:185-192. CrossRef Medline

Horak M, Chang K, Wenthold RJ (2008) Masking of the endoplasmic reticulum retention signals during assembly of the NMDA receptor. J Neurosci 28:3500-3509. CrossRef Medline

Huh KH, Wenthold RJ (1999) Turnover analysis of glutamate receptors identifies a rapidly degraded pool of the N-methyl-D-aspartate receptor subunit, NR1, in cultured cerebellar granule cells. J Biol Chem 274:151157. CrossRef Medline

Karakas E, Simorowski N, Furukawa H (2011) Subunit arrangement and 
phenylethanolamine binding in GluN1/GluN2B NMDA receptors. Nature 475:249-253. CrossRef Medline

Kawate T, Gouaux E (2006) Fluorescence-detection size-exclusion chromatography for precrystallization screening of integral membrane proteins. Structure 14:673-681. CrossRef Medline

Lee CH, Gouaux E (2011) Amino terminal domains of the NMDA receptor are organized as local heterodimers. PLoS One 6:e19180. CrossRef Medline

Lu W, Roche KW (2012) Posttranslational regulation of AMPA receptor trafficking and function. Curr Opin Neurobiol 22:470-479. Medline

Lu W, Shi Y, Jackson AC, Bjorgan K, During MJ, Sprengel R, Seeburg PH, Nicoll RA (2009) Subunit composition of synaptic AMPA receptors revealed by a single-cell genetic approach. Neuron 62:254-268. CrossRef Medline

Man HY, Lin JW, Ju WH, Ahmadian G, Liu L, Becker LE, Sheng M, Wang YT (2000) Regulation of AMPA receptor-mediated synaptic transmission by clathrin-dependent receptor internalization. Neuron 25:649-662. CrossRef Medline

Mayer ML (2011) Structure and mechanism of glutamate receptor ion channel assembly, activation and modulation. Curr Opin Neurobiol 21: 283-290. CrossRef Medline

Meddows E, Le Bourdelles B, Grimwood S, Wafford K, Sandhu S, Whiting P, McIlhinney RA (2001) Identification of molecular determinants that are important in the assembly of N-methyl-D-aspartate receptors. J Biol Chem 276:18795-18803. CrossRef Medline

Nakagawa T (2010) The biochemistry, ultrastructure, and subunit assembly mechanism of AMPA receptors. Mol Neurobiol 42:161-184. CrossRef Medline

Papadakis M, Hawkins LM, Stephenson FA (2004) Appropriate NR1-NR1 disulfide-linked homodimer formation is requisite for efficient expression of functional, cell surface N-methyl-D-aspartate NR1/NR2 receptors. J Biol Chem 279:14703-14712. CrossRef Medline

Pasternack A, Coleman SK, Jouppila A, Mottershead DG, Lindfors M, Pasternack M, Keinänen K (2002) Alpha-amino-3-hydroxy-5-methyl-4isoxazolepropionic acid (AMPA) receptor channels lacking the N-terminal domain. J Biol Chem 277:49662-49667. CrossRef Medline

Puddifoot CA, Chen PE, Schoepfer R, Wyllie DJ (2009) Pharmacological characterization of recombinant NR1/NR2A NMDA receptors with truncated and deleted carboxy termini expressed in Xenopus laevis oocytes. Br J Pharmacol 156:509-518. CrossRef Medline

Rossmann M, Sukumaran M, Penn AC, Veprintsev DB, Babu MM, Greger IH (2011) Subunit-selective N-terminal domain associations organize the formation of AMPA receptor heteromers. EMBO J 30:959-971. CrossRef Medline

Salussolia CL, Corrales A, Talukder I, Kazi R, Akgul G, Bowen M, Wollmuth LP (2011) Interaction of the M4 segment with other transmembrane segments is required for surface expression of mammalian alpha-amino3-hydroxy-5-methyl-4-isoxazolepropionic acid (AMPA) receptors. J Biol Chem 286:40205-40218. CrossRef Medline

Schüler T, Mesic I, Madry C, Bartholomäus I, Laube B (2008) Formation of NR1/NR2 and NR1/NR3 heterodimers constitutes the initial step in $\mathrm{N}$-methyl-D-aspartate receptor assembly. J Biol Chem 283:37-46. Medline

Sobolevsky AI, Rosconi MP, Gouaux E (2009) X-ray structure, symmetry and mechanism of an AMPA-subtype glutamate receptor. Nature 462: 745-756. CrossRef Medline

Stephenson FA, Cousins SL, Kenny AV (2008) Assembly and forward trafficking of NMDA receptors (Review). Mol Membr Biol 25:311-320. CrossRef Medline

Sukumaran M, Penn AC, Greger IH (2012) AMPA receptor assembly: atomic determinants and built-in modulators. Advances in experimental medicine and biology 970:241-264. CrossRef Medline

Sun Y, Olson R, Horning M, Armstrong N, Mayer M, Gouaux E (2002) Mechanism of glutamate receptor desensitization. Nature 417:245-253. CrossRef Medline

Traynelis SF, Wollmuth LP, McBain CJ, Menniti FS, Vance KM, Ogden KK, Hansen KB, Yuan H, Myers SJ, Dingledine R (2010) Glutamate receptor ion channels: structure, regulation, and function. Pharmacol Rev 62:405496. CrossRef Medline

Wo ZG, Oswald RE (1995) Unraveling the modular design of glutamategated ion channels. Trends Neurosci 18:161-168. CrossRef Medline

Yelshansky MV, Sobolevsky AI, Jatzke C, Wollmuth LP (2004) Block of AMPA receptor desensitization by a point mutation outside the ligandbinding domain. J Neurosci 24:4728-4736. CrossRef Medline 